\title{
Consumer electronics is redesigning our cars? Challenges of integrated technologies for sensing, computing and storage
}

\author{
Francesco Pieri, Cristian Zambelli, Andrea Nannini, Piero Olivo, Sergio Saponara
}

\begin{abstract}
The paper critically analyzes the trends and limits of integrated technologies for sensing, computing and data storage when devices and systems, originally developed for consumer electronics, are used for self-driving cars. Some hints, supported by theoretical analysis and experimental measures, are provided to overcome the issues of inertial sensors, micro-mirrors for Lidar scanners, car data/program memories and computing platforms.
\end{abstract}

Keywords-Driver Assistance, Sensors and Control, Navigation

\section{INTRODUCTION}

As witnessed by the 2017 editions of Consumer Electronics Show in US and Mobile World Congress in Europe, consumer electronics is changing the way of designing, producing and driving cars, including human-vehicle interfaces (HVI) and vehicle to everything (V2X) communications. IBM studies on the future of automotive industry [1] predict that the borders with consumer industry will blur and cars will be consumercentric. Technology roadmaps [2-6] predict that, by 2030, cars will be connected, automated and electrified to increase safety, energy efficiency, inclusivity and comfort. The automotive market of 80-million vehicles sold worldwide per year is suited for microelectronics and MEMS/MOEMS (micro-opto-electromechanical-system) technologies. Value in cars is shifting from chassis/powertrain to electronics and sensors (35\% of a car cost in 2025 [7]). Around $90 \%$ of all innovations in new cars are driven by electronics, see Fig. 1. Lots of consumer components can be used on-board cars, e.g.:

- inertial sensors (accelerometers and gyroscopes) to estimate the vehicle dynamics and stability, and to improve the accuracy of the position/navigation system, see Figs. 2 and 3;

- micro-mirrors for head-up display projectors, or Lidar scanning in assisted-driving;

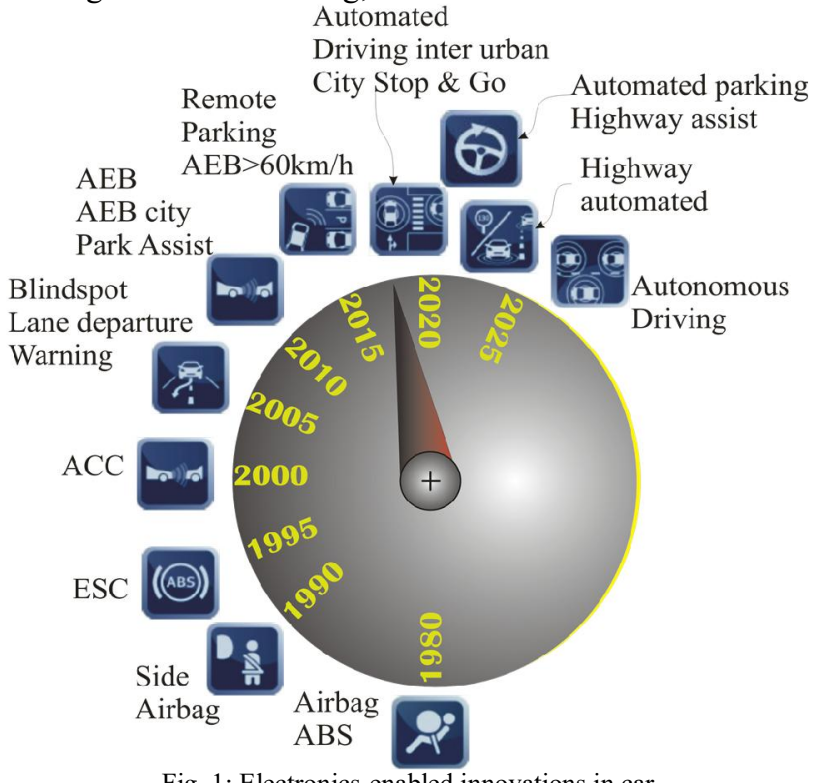

Fig. 1: Electronics-enabled innovations in car
- microcontrollers (MCU) with embedded non-volatile memory (NVM) to implement sensor fusion or control algorithms.

Vehicles require high performance components, operating in harsh environments, ensuring fault robustness, security and functional safety [8]. For most of devices, originally developed for consumer electronics, the use for self-driving is not straightforward. The issues are not only the cost, although the price of 3D Lidar is still a bottleneck [9], but mainly the performance and reliability. To this aim, this paper critically reviews the challenges of integrated components for sensing, computing and data storage, in the automotive scenario. Suggestions to overcome these issues are provided, supported by theoretical analysis and experimental measures.

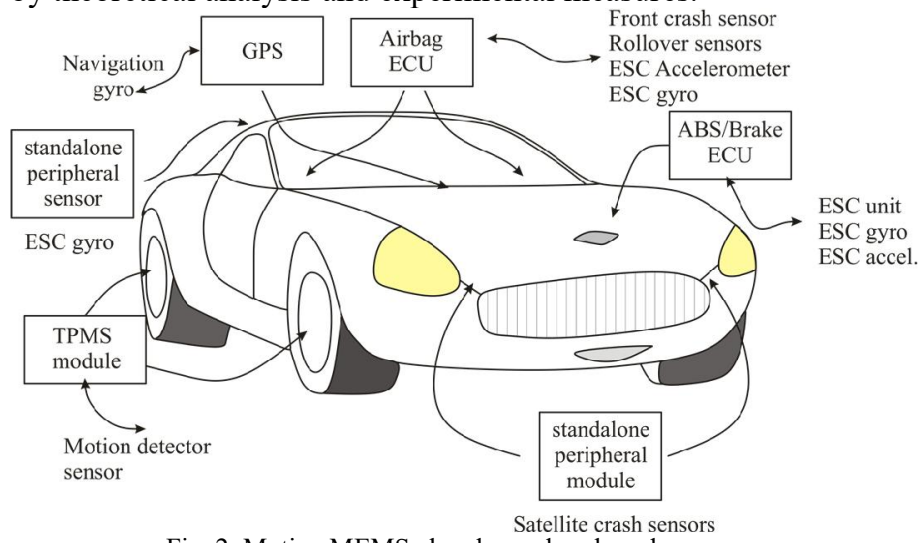

Fig. 2: Motion MEMS already used on-board cars

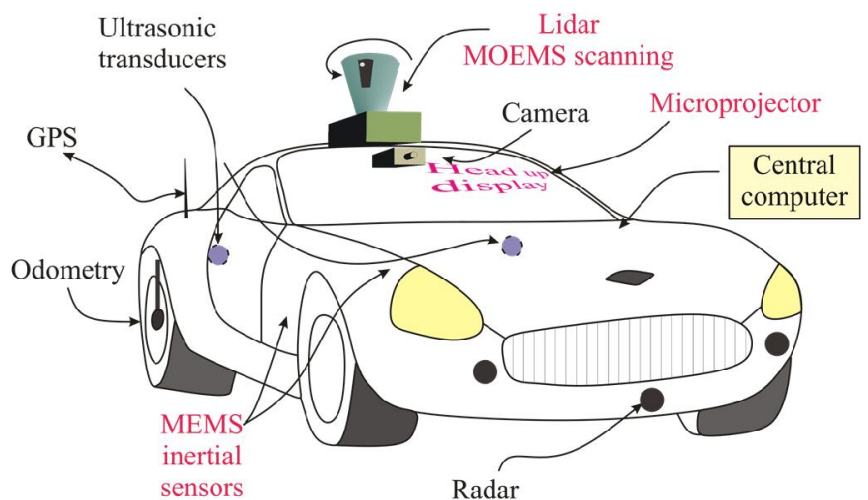

Fig. 3: MEMS/MOEMS in assisted/autonomous cars

II. INERTIAL MEMS FOR SAFETY-CRITICAL APPLICATIONS A. Automotive applications of inertial MEMS

Concerning MEMS inertial sensors, first commercial accelerometers were available by Analog Devices since the mid ' $90 \mathrm{~s}$, and commercial gyroscopes by Bosch (angular rate sensors) lagging few years later. The first automotive applications such as air-bag crash detection for accelerometers or electronic stability control for gyroscopes, were not highly demanding in terms of sensor performances. This allowed the 
development of low cost devices in high volumes. By mid '00s, low-cost, single-package, 3-axis accelerometers enabled many consumer applications, from videogame controllers to smartphone gesture recognition. Consumer applications fostered the MEMS market expansion in the following years. Today, consumer-grade MEMS IMUs (inertial measurement units, with 3-axis acceleration, 3-axis angle rate and 3-axis magnetic field measurements), such as ST LSM9DS1 or InvenSense MPU-9250 are available at a cost of a few USD. In contrast, the exploitation of inertial MEMS to some automotive applications, for which they were envisioned as enabling technology since the MEMS beginnings, has proven elusive. Among them navigation and positioning, with errors of a few $\mathrm{cm}$, are the most prominent, being essential for autonomous driving. In an Inertial Navigation System (INS) the accurate determination of position, velocity and orientation relies on the accuracy of the IMU around which the INS is built. Position and orientation are determined by time integration of IMU data. The IMU-based INS solution is typically combined with a satellite-based system [10], see Fig. 4. The INS solution is affected by errors intrinsic to MEMS sensors.

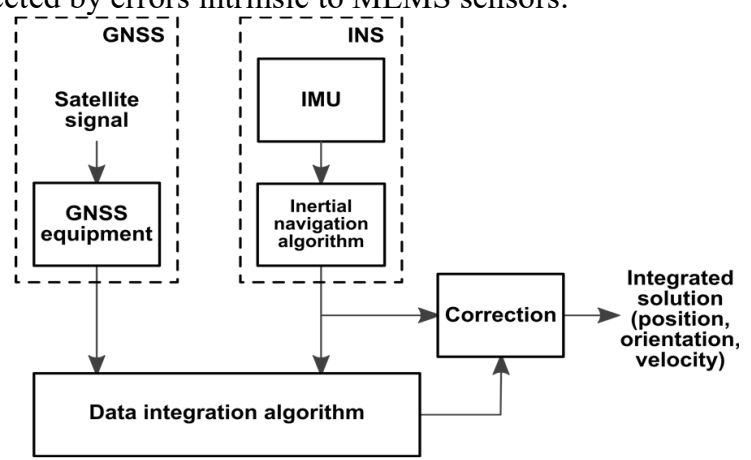

Fig. 4: Navigation system integrating satellite and INS sub-systems.

\section{B. Limits and research trends of automotive inertial MEMS}

Each sensor shows an offset (or bias), with a temperaturedependent component. Bias is given in $m g$ for accelerometers, and degrees/ $h$ for gyroscopes. Based on bias, IMUs [10] are ranked in different grades (Table 1). Highest grades cannot be reached with current MEMS technology, and are confined to high-value, low volume applications such as aerospace and defense, where a single IMU can cost tens to hundreds of thousands USD. Due to bias, the estimated position and orientation, even at rest, drifts with time. The error on the estimated position caused by accelerometer and gyroscope bias increases as the second and third power of time, respectively [10], and grows to levels unacceptable for navigation. Table 2 shows an error estimate after ten seconds for the different IMU grades, calculated based on [10]. To move current MEMS IMUs to tactical or higher grades, reliable in-run calibration techniques are mandatory [11]. Electronic calibration [12] compensates the effect of temperature on bias, and part of the effect of fabrication imperfections, for a limited increase in complexity and cost. Mechanical calibration requires periodic controlled rotation along one or more axes. Most performing solutions allow mechanical calibration in specific applications [13], but this approach is not compatible with low-cost systems. Continuous improvement of low-cost calibration techniques plays a key role in high-performance MEMS inertial systems
[11]. Bias reduction in low-cost MEMS can be also achieved through better control of process variability [14].

Inertial sensors are also affected by output random noise, expressed in terms of its noise spectral density, measured in $\mu \mathrm{g} / \sqrt{\mathrm{Hz}}$ for accelerometers, $\mathrm{deg} / \mathrm{s} / \sqrt{\mathrm{Hz}}$ for gyroscopes. Noise, even at zero input, causes the standard deviations of the estimated angles and positions to grow as a function of time as $t^{1 / 2}$ and $t^{3 / 2}$, respectively [15]. Higher noise levels are linked to higher thermo-mechanical losses in the MEMS component. However, electronic noise in the readout circuit gives an essential contribution to system noise [16].

TABLE 1. IMU GRADES BY BIAS VALUES

\begin{tabular}{|c|c|c|}
\hline IMU grade & Acceleration bias, $\mathbf{m g}$ & Angular rate bias, $\mathbf{d e g} / \mathbf{h r}$ \\
\hline Strategic & $10^{-3}-10^{-2}$ & $10^{-4}-10^{-3}$ \\
\hline Navigation & $10^{-2}-1$ & $10^{-3}-0.1$ \\
\hline Tactical & $1-30$ & $0.1-30$ \\
\hline Consumer & $>30$ & $>30$ \\
\hline
\end{tabular}

TABLE 2. TEN-SECOND POSITION ERRORS DUE TO SENSOR BIAS

\begin{tabular}{|c|c|c|}
\hline IMU grade & Due to acceleration bias & Due to angular rate bias \\
\hline Strategic & $<0.5 \times 10^{-3} \mathrm{~m}$ & $<8 \times 10^{-6} \mathrm{~m}$ \\
\hline Navigation & $0.5 \times 10^{-3}-0.5 \mathrm{~m}$ & $8 \times 10^{-6}-0.8 \times 10^{-3} \mathrm{~m}$ \\
\hline Tactical & $0.5-15 \mathrm{~m}$ & $0.8 \times 10^{-3}-0.25 \mathrm{~m}$ \\
\hline Consumer & $>15 \mathrm{~m}$ & $>0.25 \mathrm{~m}$ \\
\hline
\end{tabular}

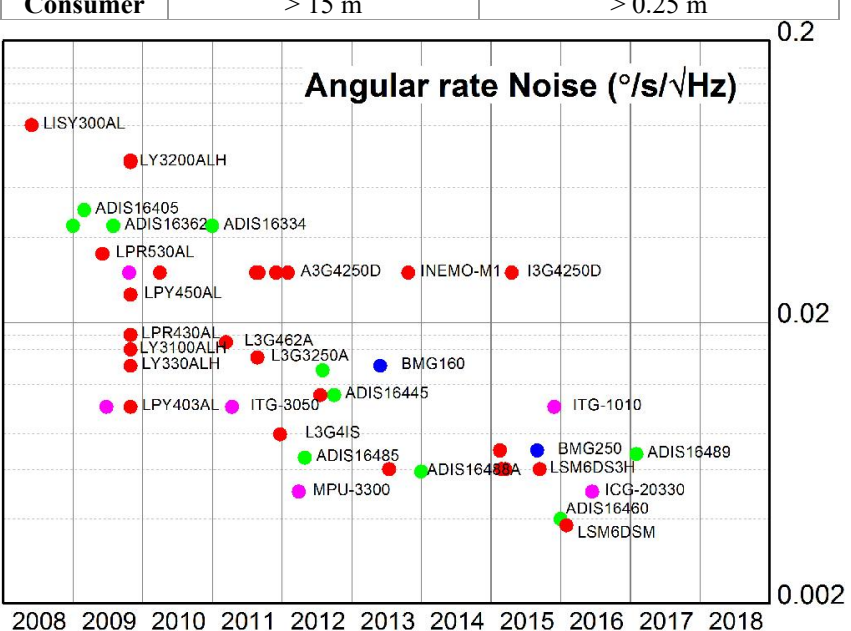

Release date

Fig. 5: Noise spectral density of several recent commercial MEMS gyroscopes/IMUs, by release date. Color marks the supplier (red = STM, green $=$ Analog Devices, purple $=$ InvenSense, blue $=$ Bosch).

At least for MEMS gyroscopes, there is evidence that the currently adopted technology, based on the tuning-fork Coriolis (TFC) principle and capacitive driving and sensing, is getting closer to what looks as a hard limit, at around and above $2 \cdot 10^{-3}$ $\mathrm{deg} / \mathrm{s} / \sqrt{\mathrm{Hz}}$ [17]. Analysis of commercial devices in Fig. 5 shows that the noise floor for consumer market gyroscopes has been close to this limit for several years now. The future roadmap to very low noise gyroscopes is currently not set, and several alternative approaches are researched. An improvement in noise floor of TFC gyroscopes has been demonstrated for piezoresistive detection [18]. However, piezoresistive sensing has issues of linearity and temperature stability. Capacitive (or piezoelectric) resonant bulk-mode gyroscopes, operating at relatively high frequency (around two decades above TFC gyroscopes, which work at tens of $\mathrm{kHz}$ ), exhibit an intrinsically lower mechanical noise, which is however, only part of the total output noise [19]. There is also research on devices based on 
precession, the Rate-Integrating Gyroscopes (RIGs), whose output is directly proportional to the angle, and not the angle rate [20]. This feature reduces the impact of bias and noise significantly, as integration of the output is avoided. RIGs are still in their infancy, and their potential is still not clear.

\section{MOEMS MIRRORS FOR LIDAR SCANNERS}

\section{A. Lidar sensing for assisted and autonomous driving}

Automotive Lidars will play a leading role as essential components in the Advanced Driver Assistance Systems (ADAS), designed to help the driver and increase car safety. The real consumer expectation, fueled by relentless market hype, is for autonomous (i.e. driverless), not simply assisted, driving, and current systems (and their sensor components) are not ready for this yet. A validation of this has been given in June 2016 by the first fatality involving a self-driving car (a Tesla model S). The Tesla system, which mostly relied on cameras and image recognition techniques, failed to identify an incoming truck. Since then, Tesla is experimenting with Radars and Lidars and many players are aggressively pursuing Lidarbased solutions. Waymo, Google's self-driving car division, sued Uber in February 2017 for allegedly stealing the technology behind Waymo's Lidar. As with most innovations in the highly-competitive car market, the issues with automotive Lidars are performance, reliability and, primarily, cost. Of the possible implementations, solid-state solutions, see Table 3, such as the ones developed at Velodyne, Quanergy, LeddarTech, ASC (acquired by Continental), are ostensibly strong on the last two points. In these systems, the IR laser beams used for obstacle detection are not scanned through the field-of-view (FOV), or are scanned with Optical Phased Arrays (OPAs) that do not require moving parts. True scanning Lidars, however, promise higher performance and flexibility. The most recent, mechanically scanned Lidar of front-runner Velodyne, whose devices were the system of choice for Google, is still rated at around 8k USD, while the automotive industry aims at a cost of few hundreds of USD, or less.

\section{B. Micro-mirrors for low-cost Lidar scanning}

MOEMS scanning micro-mirrors can replace the motorized, mechanical Lidar version at a fraction of the cost. While several such solutions are under development, none of them has hit the market yet. Among design specifications for Lidars, a large angular FOV is desirable to reduce to numbers of Lidars to explore the full 360-degrees around the vehicle. While a single, 360-deegres rotating mirror can be used to achieve all-around view, such a system requires a bulky and esthetically unappealing roof mount to raise the device and avoid blind angles where the vehicle body shadows its surroundings. The essential advantage of a mechanically scanned beam with

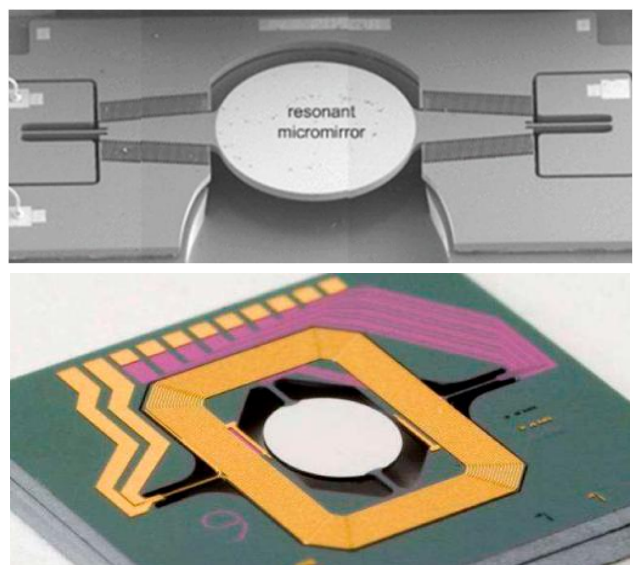

Fig. 6: (top) 1D electrostatic micro-mirror (STM, from [23]) and (bottom) 2D magnetic gimbaled micro-mirror (Microvision Inc., from [24]).

respect to (more cost-competitive) solid-state scanners is the wider scanning angle. In single-axis MEMS micro-mirrors, angular deflection is obtained with oscillating motion of the (deformable) mirror suspensions, which act as torsional springs (Fig. 6, top). Mirrors with true 360-degrees scanning range have been developed [25], where a clever 2D actuation kinematics, based on asymmetric suspensions, gives a full rotation around the axis of the incident laser beam. Two-axes (2D) MEMS micro-mirrors are also actively developed, but their main envisioned application is currently for image projectors, where a raster scanning pattern is used [26]. 2D mirrors typically include a gimbal (Fig. 6, bottom) to mechanically decouple the two rotation axes, and so their structure is complex and bulky, limiting the maximum operating frequency. More compact, gimbal-less solutions are in development, but mechanical interference between axes can be a problem. In automotive Lidars, the requirement for vertical FOV is more limited than for horizontal FOV. For these reasons, expansion of the vertical FOV can be obtained by a linear, vertical array of micromirrors, each with its own beam, instead of a single 2D mirror. To maximize the oscillation angle, mirrors can be operated at or near their mechanical resonance frequency. In this regime, the deflection is proportional to the mechanical quality factor $Q$. To boost the $Q$, and with it the scanning range, operation in vacuum is often used, as the elimination of drag of air results in an orders-of-magnitude increase. Vacuum packaging, however, comes with its problems of cost and reliability [27].

Another crucial parameter for MEMS mirrors is their size. The mirror diameter directly impacts the collected laser power, and thus the maximum achievable range. Fully automated driving requires a range higher than $100 \mathrm{~m}$. Large diameters come with a series of technology drawbacks. Among them: mirror

TABLE 3. SAMPLE DECLARED PERFORMANCES OF KNOWN LIDAR SOLUTIONS.

\begin{tabular}{|c|c|c|c|c|c|c|c|}
\hline Supplier & Type & HFOV in deg & VFOV in deg & Scanning Freq. & Cost & Range & Source \\
\hline Osram/Infineon/Innoluce & Scanning, MEMS & $120,($ res. 0.1$)$ & 20 (res. 0.5$)$ & $<2 \mathrm{kHz}$ & 40 USD & $200 \mathrm{~m}$ & [21] \\
\hline Quanergy & Scanning, OPA & 120 & 120 & $\mathrm{~N} / \mathrm{A}$ & 250 USD & $150 \mathrm{~m}$ & [22] \\
\hline Velodyne (VLP-16) & Scanning mechanical & 360 (res $0.1-0.4)$ & 30 (res. 2) & $5-20 \mathrm{~Hz}$ & 7999 USD & $300 \mathrm{~m}$ & Velodyne site \\
\hline LeddarTech (LeddarVu) & Solid-state & 100 & $0.3-3$ & $\mathrm{~N} / \mathrm{A}$ & 750 USD & $60 \mathrm{~m}$ & datasheet \\
\hline ASC (Peregrine) & Solid-state & up to 60 (res. 0.5$)$ & up to 15 (res. 0.5 ) & $20 \mathrm{~Hz}$ & $\mathrm{~N} / \mathrm{A}$ & $\mathrm{N} / \mathrm{A}$ & ASC website \\
\hline Microvision(PSE-0400Li-101) & Scanning MEMS & 90 (res. 0.18) & 30 (res. 0.08) & $30 \mathrm{~Hz}$ & $\mathrm{~N} / \mathrm{A}$ & $15 \mathrm{~m}$ & Microvision \\
\hline
\end{tabular}


actuation is difficult because of the larger deflections involved; it is harder to achieve a good optical flatness, which is required to avoid deformation of the beam. In gimballed 2D mirrors, these problems are exacerbated. Published scanning micromirror diameters typically range from $2 \mathrm{~mm}$ up to $7 \mathrm{~mm}$ [25]. At this size, resonance frequency scale from several tens of $\mathrm{kHz}$ to $1 \mathrm{kHz}$ or less. For successful long-range MEMS Lidars, increase of the mirror size is probably one of the decisive issues.

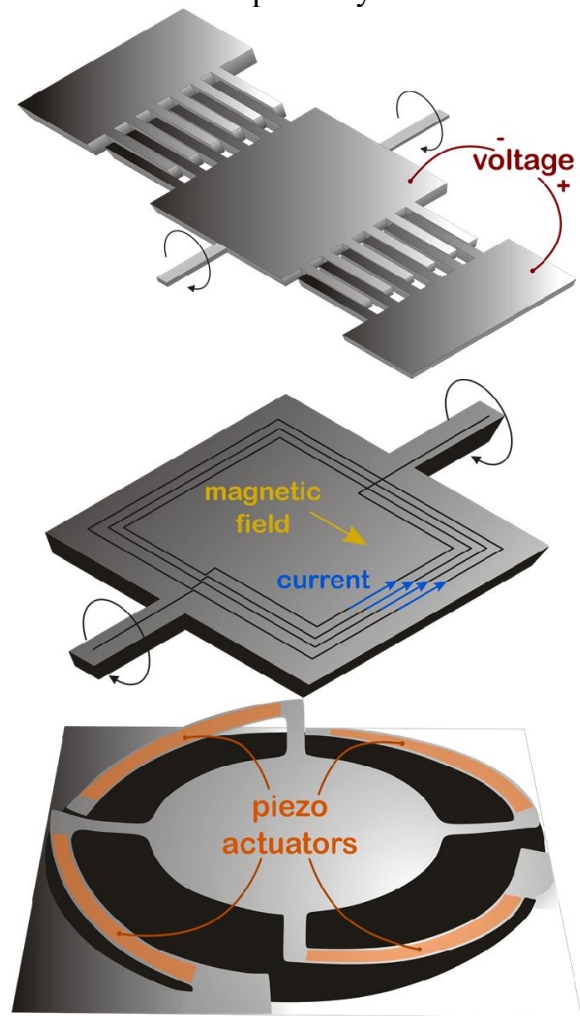

Fig. 7: structure of micro-mirrors with three different actuations schemes: (top) electrostatic [23], (center) magnetic [28], (bottom) piezoelectric [29].

The type of actuation has also an impact on the mirror structure and performances. For "standard" MEMS, the simple and wellestablished electrostatic actuation has normally been the way to go. In this approach, deflection is obtained by applying a voltage between two plates, one of which is movable and attached to the mirror. Electrostatic actuation, however, does not scale well with mirror size. To increase the torque without increasing the actuator size, the plates are made of dozens (or hundreds) of tiny interdigitated structures or "comb fingers," (Fig. 7, top) [23]. Despite this, actuation voltages of tens or even hundreds of volts are not uncommon [26]. Magnetic actuation, where a torque is generated by an external magnetic field over a current flowing into coils placed on the mirror surface (Fig. 7, center) [28], is characterized by low voltages but comparatively high currents (tens to hundreds of milliamps). The coil increases the mirror size, and the problems connected with it, and external magnets are required. A third, interesting but less pursued actuation scheme is based on piezoelectric actuators (Fig. 7, bottom) [29], which promise large torques at reasonable voltage levels. The main drawbacks are the complex kinematics required to amplify the modest deformations of piezoelectric materials, and the integration of piezoelectric materials into a MEMS technology. The latter is on its way to be solved, also because piezoelectrics is an enabling technology for other applications. As of today, it is far from clear which the technical path to success for automotive Lidars will be. What seems likely, however, is that the requirements of high performance, small dimensions, and, above all, mass-market compatible costs, will force a path that goes through the development of high-performance MEMS micro-mirrors.

\section{HIGH-RELIABLE NVM FOR AUTOMOTIVE ELECTRONICS}

\section{A. Automotive applications of NVMs}

Automotive electronics applications require storage devices dependently on the target functionality to be implemented. Such a feature can be achieved through the integration of a NVM. As shown in Fig. 8, there are two main segments that involve different NVM technologies: the infotainment (i.e., the plethora of navigation, in-car entertainment, connectivity applications), and the in-vehicle embedded systems to be designed either for sophisticated control algorithms or sensor data processing.

Concerning the data storage for infotainment applications, the choice of many automotive vendors lies on NAND Flash. This mature consumer NVM technology is characterized by a very high storage density (in the terabits domain) with a very low cost per bit, easily achievable by implementing the multi-bits per cell storage paradigm [30]. This solution is favored for mass storage applications where a high amount of data need to be stored, often trading with the poor write/read access performance and the inherent reliability of the media. The preferred format for frequent user data exchange is the Secure Digital (SD) card through its many variants (e.g., SD, miniSD, microSD, etc.), which integrate: a controller for protocol interfacing and basic memory management functions, and a NAND Flash memory module. The media removability feature is of great benefit since it allows off-vehicle data updating. Advancement in automotive shows that this NVM technology can be easily integrated, along with a dedicated memory controller, to form complex storage systems like eMMC [30] and Solid State Drives [31]. As said, the reliability constraints for this memory technology is the same as for any consumer application embodying a NAND Flash since no hostile operating conditions are foreseen in terms of operating temperature, voltages, and other electrical concerns. From the qualifications and reliability standpoint those memories are characterized by a very limited lifetime especially for multi-bits products (usually few years) with capability to retain the information of few months (i.e., 3 months at a storage temperature of $40^{\circ} \mathrm{C}$ ) [32].

Concerning the in-vehicle embedded systems scenario for NVM, the considerations on performance, reliability, and technological design, change radically from previous context.

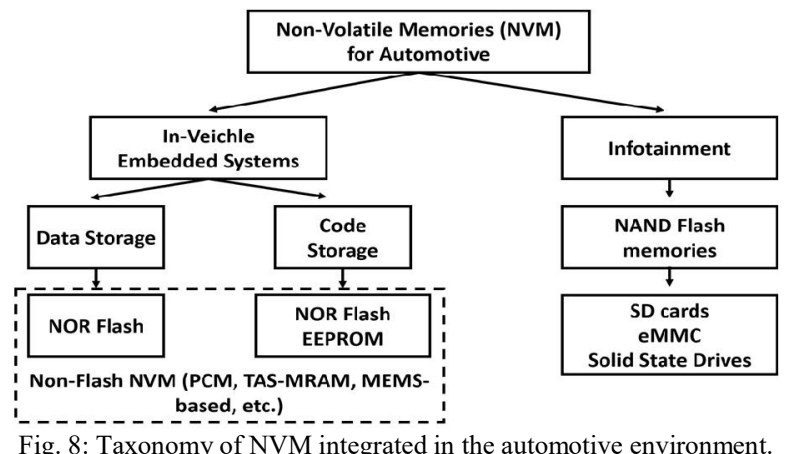


As pointed out in Fig. 8, there are two main application branches for embedded memories: data storage and code storage. The data storage class is intended for all the sensor calibration, V2X, or simple data logging activities in the car that do not require an excessive amount of data, whereas the code storage class is intended for real time operating systems executing heavy computation algorithms or sensor/actuator control codes execution. In both cases there is no need for mass storage platforms, therefore all these NVMs should have a storage density ranging from few kilobits up to some megabytes for computation-hungrier algorithms. However, it must be pointed out that the increase of the number of sensors integrated in the car, as well as the number of functionalities that require powerful integrated microcontrollers with larger word size (from 8 up to 64 bits) will call in a nearby future for a storage density increase. Fig. 9 shows the trend relationship between the NVM size and the computational power in MIPS for automotive embedded MCUs [33]. As evidenced by the figure, both parameters are expected to increase at the same pace in the next years, reaching tens of $\mathrm{MB}$ in NVM size for a corresponding computational power of few kilo-MIPS in 2020. It must be pointed out that the data in the figure refers to assisted driving scenario and active safety applications that are far from the requirements for autonomous driving as pointed out in [34]. For these NVM, the reliability becomes at the forefront since they could control critical applications for the vehicle powertrain or safety features like airbags and collision avoidance systems. Generally, the desired defectivity rate for these memories must be below $1 \mathrm{ppm}$.

\section{B. NVMs operating in harsh environments}

One of the biggest issues for embedded NVM is related to the high temperatures, up to $200^{\circ} \mathrm{C}$, reached in some under-thehood worst-case scenarios that severely threaten the nonvolatility of the stored data. The effect of the High Temperature on Data Retention (HTDR) is shown in Fig. 10. The discrimination of the stored data content is achieved by comparing a physical quantity (e.g., a threshold voltage or a read current) against a reference level (the read level) that is placed between the cells distribution of logical ' 1 ' (i.e., the program state) and logical ' 0 ' (i.e., the erase state). The application of a high temperature on the memory causes a degradation of the physical properties of the cells, thus shifting the distributions close to each other, with some cells in the distribution tails erroneously crossing the read level (causing a bit-flip or a data corruption). Typical consumer NVMs are designed in the front-end of the semiconductor process and can

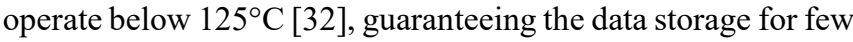
years at that temperature only for a very low write cycling count (i.e., the measure of the memory lifetime wear-out due to repeated data writing and erasing). Therefore, a special memory design must be performed to work in automotive environments. Usual design solutions incorporate single-bit per cell storage paradigms (in some cases even differential redundancy is applied [35]) coupled with robust CMOS technologies that are quite far from state-of-art scaled consumer products. Nonetheless, all the peculiarities of the hostile automotive environment must be taken into account in the overall reliability computation. Care must be taken against vibrations, mechanical stresses, and induced electromagnetic issues due to high currents flowing in some actuators, that could modify, once again, the retention features of the stored data. Embedded solutions based on the EEPROM concept have been developed in the mid '00s to cope with the temperature issue [36]. Multikbits memory modules functionality, and data retention of over 30 years for the automotive temperature range, have been achieved by a dedicated cell design in a $0.35 \mu \mathrm{m}$ process [34].

NVM for assisted driving scenario or safety applications

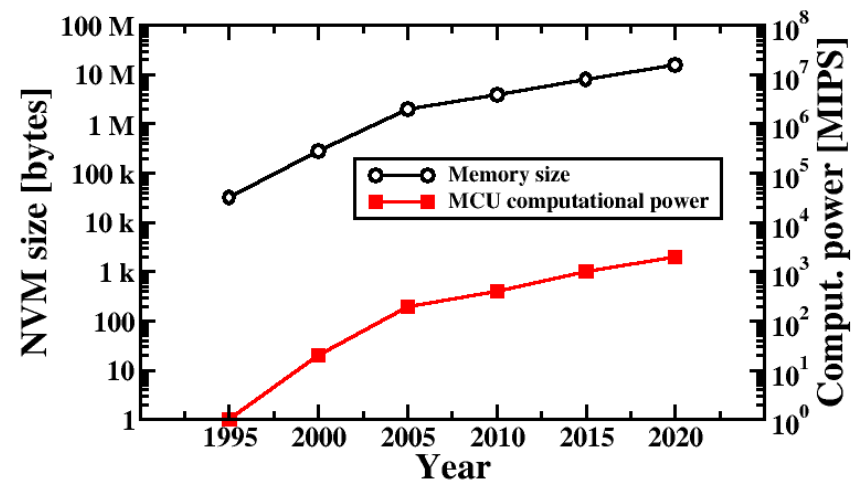

Fig. 9: Trend of embedded NVM size compared to the required computational power for assisted-driving or active safety automotive applications [33].

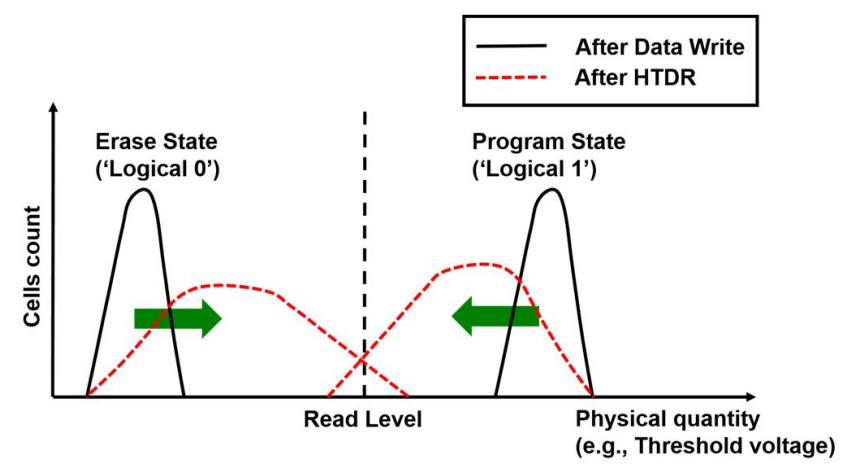

Fig. 10: Effect of the High Temperature Data Retention (HTDR) on the stored data. The data corruption occurs in the tail cells crossing the read level.

Moreover, write cycling of over $200 \mathrm{k}$ writes, tested up to $180^{\circ} \mathrm{C}$, has been demonstrated, proving the suitability in all applications requiring frequent data manipulation and storage (e.g., engine start/stop systems). The drawbacks of these solutions lie on the write and erase times (i.e., few milliseconds) and on the limited storage density. In particular, the latter point is critical to limit the cost per bit as much as possible. In such landscape NOR Flash became the de facto storage medium both for code and data applications in automotive embedded systems. Attractive features of this technology are the low read access latency (being in the hundreds of nanoseconds range) and a relatively long endurance of the storage medium, that is diversified in relation to the memory application that could be pure code in-place execution or data storage [33]. Data storage NOR Flash generally exhibits a higher endurance (up to $500 \mathrm{k}$ write cycles) to cope with the new requirements dictated by new ECU (Electronic Control Unit) applications like gas emission control or start/stop systems. Among the several technology variants that can be integrated (i.e., 1T-NOR, 2T-NOR, splitgate, etc.), the Fully Fowler-Nordheim (FN/FN) concept stands out due to its lower energy consumption required in the programming operation and a relative ease of integration for large density products [33]. However, from the pure reliability 
viewpoint, the erase operation has always been an issue for every NOR Flash technology so far [37]. In the last decades, special emphasis has been put on the reduction of the standard erase failure mechanisms by implementing either proper correction algorithms or failure screening techniques aiming at a memory fail rate reduction below $1 \mathrm{ppm}$ [38]. Such activity involves: complex time-consuming recovery algorithms that burden on the erase latency, Error Correction Codes (ECC) design for fast error detection and correction to limit the impact on the read latency, and redundancy-based solutions that increase the costs of the final product where the NVM is embedded. An additional item that arose as a potential limiting factor for state-of-the-art NVM in code/data storage for automotive is the scalability of the memory bitcells that is function of the technology node used in the product integration [33]. Due to the severe constraints imposed in terms of data retention after storage, it is impossible to scale the dimensions below a certain limit, therefore classical EEPROM/Flash cells cannot shrink in a cost-effective manner. To this extent, innovative memory cell concepts integrated in the Back-EndOf-Line (BEOL) or standard CMOS processes, are explored in order to overcome the scaling barrier while improving the $\mathrm{read} /$ write performance of the overall embedded NVM subsystem [39]. All the emerging memory concepts stem from the charge storage paradigm to interpret the information, and therefore base on radically different physical principles.

Phase Change Memories (PCM) are the first emerging concept studied to effectively replace NOR Flash. The operating principle of a PCM device relies on the phase transformation of a small volume of phase change material (usually a chalcogenide compound) between an amorphous phase (high resistivity) and a crystalline phase (low resistivity) [40]. The contrast in conductivity between the two phases allows discriminating the stored information. The principal advantages of this technology are: the low programming voltages, the very short reading and programming times, the good shrinking prospects and the low manufacturing cost due to the reduced number of required masks levels [41]. However, there are some limiting factors in terms of data retention that currently do not candidate this memory for automotive use. Materials research is exploited to find adequate solutions to this problem [40].

An interesting non-charge based NVM concept for automotive environment has been studied with MEMS-based memory cells. The working principle of a single memory element bases on a teeter-totter MEMS element [42]. When applying a voltage on the pull-in electrode (the program electrode) an electrostatic force will tilt the plate to touch the right contact electrode. The stiction forces at this contact point will keep the floating plate in this position. While in this position, a voltage can be applied on the left pull-in electrode (the erase electrode), resulting in an electrostatic force on the left side of the floating plate. When this electrostatic force reaches a threshold value, it will overcome the stiction forces at the right contact electrode and the plate will tilt to the left and land on the left contact electrode. The advantages of this memory technology are a very good data retention at extreme temperature and a relative low operational power. Unfortunately, the overall area occupation of the MEMS element is still too much to be integrated in multi-megabit embedded NVM products. Recently, Resistive RAM (RRAM) and Conductive Bridge RAM (CBRAM) gained interest as embedded products in a microcontroller unit [43]. This technology uses a dielectric or a chalcogenide, which is normally insulating, but can be made conductive through a filament or conduction path formed after the application of a sufficiently high voltage [41]. Considerable progress has been made in device integration as well as in understanding the physical/chemical properties of the resistance change behavior. Although memory arrays in the literature demonstrated excellent performance parameters [44], the inter-cell variability (variations between cells) and intra-cell variability (cycle-tocycle variations of any given cell) still prevent RRAM manufacturing from fast commercialization [45]. Several limits appear on the temperature sensitivity of the storage medium. Magnetic Random Access Memories (MRAM) are one of the most promising candidates to replace traditional Flash in future non-volatile memories generations [46]. Among the MRAM paradigms that are under investigation, the Thermally Assisted Switching represents a good candidate for a replacement of the standard embedded flash memories in harsh environments [46]. The information storage mechanism is based on the currentinduced magnetization switch of a magnetic material: dependently on the imposed field direction with respect to that of a reference layer, a defined resistance of the material can be achieved. The reliability and the cell-to-cell variability during the typical automotive tests (i.e., $500 \mathrm{k}$ write cycles and $200^{\circ} \mathrm{C}$ data retention targets) have been evaluated on $1 \mathrm{kbits}$ arrays manufactured with different back-end processes and proven achievable [47]. Despite the excellent performance metrics displayed in terms of read/write speed, there are concerns in terms of power consumption and high cost per bit due to the large amount of materials requested to manufacture the memory cells. Table 4 summarizes the benchmarks of the characteristic parameters for each embedded NVM technology starting from the state-of-the-art up to the innovative concepts.

\section{AUTOMOTIVE MCUS AND COMPUTING PLATFORMS}

The high computation needs of automated and connected cars, see Fig. 11, is revolutionizing the scenario of automotive MCUs

TABLE 4. BENCHMARK OF DIFFERENT EMBEDDED NVM TECHNOLOGIES FOR AUTOMOTIVE ENVIRONMENT (DATA FROM [33, 39, 42, 46]).

\begin{tabular}{|c|c|c|c|c|c|c|c|}
\hline Parameter & EEPROM & $\begin{array}{c}\text { NOR Flash } \\
\text { Code Storage }\end{array}$ & $\begin{array}{c}\text { NOR Flash } \\
\text { Data Storage }\end{array}$ & PCM & MEMS-based & $\begin{array}{c}\text { RRAM } \\
\text { CBRAM }\end{array}$ & TAS-MRAM \\
\hline Endurance & $500 \mathrm{k}$ & $10 \mathrm{k}-100 \mathrm{k}$ & $500 \mathrm{k}-1 \mathrm{M}$ & $>1 \mathrm{M}$ & $>1 \mathrm{M}$ & $100 \mathrm{k}$ & $>1 \mathrm{M}$ \\
\hline Data Retention & $>10 \mathrm{yrs} / 125^{\circ} \mathrm{C}$ & $10 \mathrm{yrs} / 125^{\circ} \mathrm{C}$ & $>10 \mathrm{yrs} / 125^{\circ} \mathrm{C}$ & $10 \mathrm{yrs} / 85^{\circ} \mathrm{C}$ & $>10 \mathrm{yrs} / 125^{\circ} \mathrm{C}$ & $10 \mathrm{yrs} / 85^{\circ} \mathrm{C}$ & $>10 \mathrm{yrs} / 125^{\circ} \mathrm{C}$ \\
\hline Power consumption & Low & Low & Low & High (Write) & Low & Low & High \\
\hline Read Latency & $20-50 \mathrm{~ns}$ & $<20 \mathrm{~ns}$ & $<20 \mathrm{~ns}$ & $>20 \mathrm{~ns}$ & $>100 \mathrm{~ns}$ & $>20 \mathrm{~ns}$ & $50-100 \mathrm{~ns}$ \\
\hline Cost per bit & Medium/High & Medium & Medium & Low & High & Low & High \\
\hline
\end{tabular}


and computing platforms. The state-of-art can be grouped in low-power MCUs, 32b MCUs for general purpose on-board operations and Artificial Intelligence (AI) autonomous cars platforms. The first two classes target a temperature range -40 to $150{ }^{\circ} \mathrm{C}$. All classes support, with different security levels, dedicated coprocessors for cybersecurity.

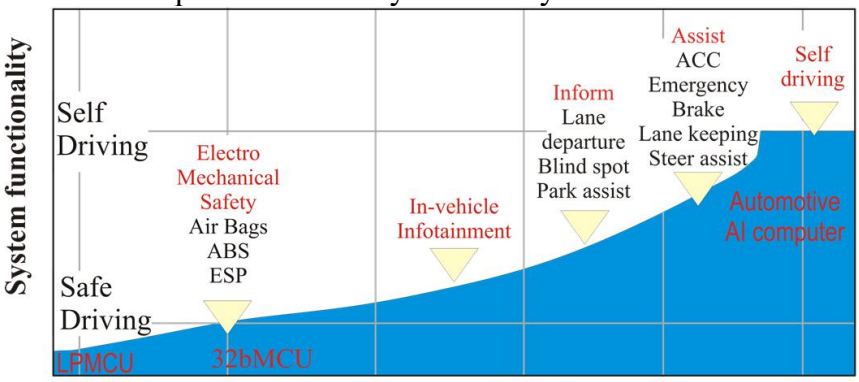

10 MIPS 100 MIPS 1GFLOPS 10GFLOPS 100GFLOPS 1TFLOPS

Fig. 11: Computation needs vs. autonomous/assisted driving functions [6]

Low-power MCUs are used for simple control and sensor/ actuator interfacing tasks. As a representative the STM8A family, in $130 \mathrm{~nm}$ EEPROM technology, has 8b Harvard 3stage pipelined single-core architecture and offers up to 20 MIPS at $24 \mathrm{MHz}, 10 \mathrm{~b}$ on-chip ADC and 2 comparators, 2 to $128 \mathrm{kB}$ program NVM plus 2 to $6 \mathrm{kB}$ data RAM. The current consumption is few $\mu \mathrm{A}$ in stand-by and $125-500 \mu \mathrm{A} / \mathrm{MHz}$, with a supply from $1.65 \mathrm{~V}$ to $5.5 \mathrm{~V}$, in operating mode. The interfaces include LIN, SPI, UART, I2C, CAN at data-rates below $1 \mathrm{Mb} / \mathrm{s}$. Today, 32b MCUs are dominating the market for active safety and advanced X-by-Wire control tasks due to their good tradeoff between computational capability and functional safety (up to ASIL-D). As example, the ST SPC5 and Freescale MPC56 families, in $90 \mathrm{~nm}$ down to $40 \mathrm{~nm}$ technology, are built on $32 \mathrm{~b}$ PowerPC instruction set, single or up to 4 cores (with dual lockstep approach), SIMD capable floating point unit, with up to 8 $\mathrm{MB}$ of embedded Flash, multi-channel 12b ADC, interfacing data-rate up to $10 \mathrm{Mb} / \mathrm{s}$ with Flexray, I2C, LIN, CAN, SPI. Similarly, the Infineon Aurix, integrated in more than 50 brand cars, ranges from a $300 \mathrm{MHz}$ triple-core device with 720 MIPS and $8 \mathrm{MB}$ of embedded Flash downto an $80 \mathrm{MHz}$ single-core with 130 MIPS and $0.5 \mathrm{MB}$ embedded Flash.

In terms of ECUs connectivity, the recent trend is increasing the in-vehicle networking data-rate with 1 and $10 \mathrm{~Gb} / \mathrm{s}$ Ethernet links, which are already adopted in the INTEL Go automotive platform. With respect to consumer applications of Ethernet, invehicle networking needs stringent deterministic behavior and hard real-time constraints. To this aim, the Ethernet TSN (Time Sensitive Network) standard initiative [48] has been recently started, which ensures that all units participating in real-time communication have a common understanding of time (time synchronization), adhere to the same rules in processing and forwarding communication (scheduling and traffic shaping), adhere to the same rules in selecting communication paths and in reserving bandwidth and time slots, utilizing more than one simultaneous path to achieve fault-tolerance.

Next-generation autonomous cars require that the whole perception process be qualified at ASIL-D level according to the ISO 26262 functional safety standard. This can be achieved by performing redundant computations with possibly dissimilar implementation techniques on two or more safe High
Performance Computing (HPC) units, which apply parallel computing and directly access high-bandwidth sensors (e.g. Radar, Lidar, camera) through LVDS or Ethernet links. Each of these HPC units should be qualified at least at ASIL-B, by implementing a range of safety mechanisms such as ECC in memory, parity and/or cyclic redundancy checks in caches and in on-chip networking infrastructure (NoC). The redundant results can be then compared by a safe micro-controller qualified for ASIL-D, which monitors the computations and decides whether the results can be trusted. The supervising micro-controller will be connected to the car backbone with run-time environment compliant with Classic Autosar Platform. From a SW point of-view implementing the Classic Autosar Platform ensures the highest real-time and safety capabilities, taking over functionalities which need response times in the lower $\mu \mathrm{s}$ domain, as well as safety monitoring capabilities in the HPC domain. Currently specified and developed, the Autosar Adaptive Platform [49] will become the automotive standard for HPC automotive units. Since the service-oriented network protocols are the same in the Classic and Adaptive platform, inter-operability between the two is given. The Autosar Adaptive Platform defines a service oriented middleware as well as system health monitoring for automotive HPC ECUs, which can run on POSIX PSE51 compatible operating systems. A common requirement for HPC ECUs is the strict separation of specific SW domains, so that in a mixed-criticality environment it can be easy to separate SW functions with different ASIL. Since the Autosar Adaptive Platform is available on Linux, this opens the door to the world of Linux-based infrastructure SW. Fig. 12 shows autonomous car SW architecture with Autosar Classic/Adaptive platforms. From an HW point-of-view for the safe micro-controller, already available $32 \mathrm{~b}$ cores, like Infineon Aurix, can be adopted. Instead, for the HPC units, massively parallel platforms are appearing in the car's market. Mass production of the Renesas R-Car H3 in $16 \mathrm{~nm}$ technology is expected in 2018 [50]. R-Car H3 includes 9 ARM's Cortex cores (8 64b A57/A53 engines with L1/L2 cache plus a $32 \mathrm{~b}$ R7 with L1 cache), offering 40k MIPS plus a PowerVR GX6650 graphics engine with 192 ALU cores for 3D graphics (more than 100 GLOPS and $4 \mathrm{~K}$ video display/streaming) and dedicated video coprocessors (H.26x/MPEGx codec, distortion compensator, IMP-X5 image recognition). The R-Car H3 is ASIL-B and has a rich set of high-rate interfaces such as Ethernet, USB, DVD/blue-ray SATA, SD card, Audio/video I/O, beside I2C and CAN. The power consumption amounts to tens of Watts. While Renesas, ST, Freescale, Infineon, and others, are classic automotive-world suppliers, new actors are entering this application domain like Nvidia and Intel, to bring on-board TFLOPS capability and AI technologies with multi-chip vehicular supercomputers. They have signed core-partnerships with mass market car' makers, Nvidia with Audi, Intel with BMW, to have on the roads AI cars by 2020 [51]. At the CES2017 the NVIDIA's CEO presented the Xavier AI car's computer, which features 30 TFLOPS capability for a power consumption of $30 \mathrm{~W}$ thanks to 8 ARM $64 \mathrm{~b}$ cores plus a 512core Volta GPU, a Video Processing Unit supporting $8 \mathrm{~K}$ video decode and encode and High Dynamic Range, as well as a computer vision accelerator. The Xavier AI is fabricated in 16 $\mathrm{nm}$ TSMC FinFET technology with an estimated complexity of 
7 billions of transistors. Intel Go is the new brand for automotive. A first multi-chip platform is available using an Aurix ASIL-D 32b MCU enhanced for computation capability by an ATOM C3000 core in $14 \mathrm{~nm}$ technology, and by Arria 10 FPGA. The letter includes an embedded dual-core $1.5 \mathrm{GHz}$ ARM A9 core, more than $1 \mathrm{M}$ logic elements (a 64b 6-LUT with $4 \mathrm{FFs}$ at the output), and $1.7 \mathrm{M}$ user flip-flops, and $64 \mathrm{Mb}$ of embedded memory. The Arria 10 family includes hardened single-precision IEEE 754 floating point, with an aggregate throughput of 1.3 TFLOPs. This platform supports level 3 automation, in which the system performs the driving task but a human driver will intervene when requested. Another INTEL platform is announced using more powerful Xeon processors, and using two multi-chip boards connected with a 16-port 10 Gb Ethernet, to sustain level 4 high automation, in which the system can perform the driving task without human intervention, and level 5 full automation, in which the system takes over all aspects of driving full time.

The power consumption of such HPC automotive platforms, will be in the range from tens to hundreds of Watts, e.g. from $30 \mathrm{~W}$ of Xavier chip to $500 \mathrm{~W}$ of the 320 TFLOPS Drive PX Pegasus board announced at GTC Europe 2017 [52]. Due to the high environmental temperature of under the hood car's electronics, passive cooling systems are not enough. Hence, the design of low-cost/low-size active cooling systems for HPC ECUs is a new emerging challenge.

To ensure high bandwidth wireless connectivity (e.g. for realtime HD map download and infotainment, over-the-air diagnostic and SW update, sensor-data upload from the vehicle for machine learning) two solutions can be adopted [53, 54]: IEEE $802.11 \mathrm{p}$ or Cellular-V2X. IEEE $802.11 \mathrm{p}$ uses $10-\mathrm{MHz}$ channels within the $(5.85-5.925 \mathrm{GHz})$ band to achieve data rates of several $\mathrm{Mb} / \mathrm{s}$ for V2X. IEEE $802.11 \mathrm{p}$ transceivers are already available in the market (e.g. STM-Autotalks chipset) and, as we discussed in [53], they can be implemented at lowcost in mature and already automotive-qualified CMOS technologies. With $33 \mathrm{dBm}$ of effective isotropic radiated power, a single-hop connectivity up to $1 \mathrm{~km}$ can be achieved. Cellular-V2X connectivity can be achieved with multilayer MIMO transmission according to emerging $5 \mathrm{G}$ transceivers. Operating both in sub- $6 \mathrm{GHz}$ and $28 \mathrm{GHz}$ millimeter wave (mmW) bands, data-rates up to several $\mathrm{Gb} / \mathrm{s}$ can be achieved. However, high-end technology nodes are required to sustain $\mathrm{mmW}$ and massive MIMO 5G operations. Moreover, the way to achieve low-latency guaranteed performance is still an open issue. A first $5 \mathrm{G}$ modem has been announced by Intel at last CES 2017, although its automotive qualification is still ongoing and the $5 \mathrm{G}$ standardization is still not frozen.

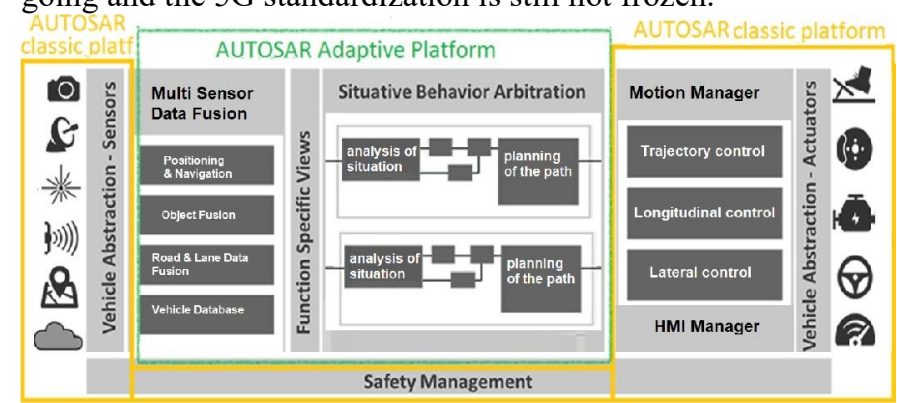

Fig. 12: Autonomous car architecture with Autosar platform (Elektrobit)

\section{Conclusions}

Consumer electronics is redesigning our cars, which will be automated, connected and shared. Several challenges have still to be won in terms of integrated technologies for sensing, computation and storage. Although for some technologies, e.g. Lidar, the cost is still a bottleneck, for other technologies diffused in consumer electronics, like MEMS sensors or NVM data storage, the main issues are noise/bias performance and reliability when operating in hash environment. Reaching high safety and security levels is also an open issue for computing platforms. While state-of-art MCU market is dominated by $32 \mathrm{~b}$ architectures, new self-driving services need the development of multi-core AI supercomputers with TFLOPS capability.

\section{About The AUThors}

F. Pieri (francesco.pieri@unipi.it) is Assistant Professor of Microelectronic Technologies at University of Pisa, His research interests include MEMS/MOEMS design and modeling, MEMS resonant sensors, and microfabrication.

P.Olivo(piero.olivo@unife.it) got MSc and PhD degrees in electronic engineering from the University of Bologna, Italy, in 1980 and 1987, respectively. He is Full Professor of Electronics with the University of Ferrara, Italy, since 1994. His research interests include the physics, reliability and characterization of innovative NVMs and SSDs.

C.Zambelli (cristian.zambelli@unife.it) got MSc and PhD degrees in electronics engineering from the University of Ferrara, Italy, in 2008 and 2012, respectively. He has been Assistant Professor with the Università of Ferrara, since 2015. His research interests include the characterization, physics, and modeling of NVMs and SSDs reliability. A. Nannini (andrea.nannini@unipi.it) got MSc and PhD degrees in 1982 and 1987, respectively. Since 2000 he is Full Professor at University of Pisa with teaching duties and research interests in Sensor and Microsystem Design, MEMS and Solid State Electronics.

S.Saponara (sergio.saponara@unipi.it) is Full Professor of Vehicular Electronics at University of Pisa, Italy and CTO of Ingeniars srl. He is IEEE Distinguished Lecturer and AE of IEEE and Springer journals, including IEEE CEM.

\section{REFERENCES}

[1] B. Stanley et al., "A new relationship - people and cars: how consumers around the world want cars to fit into their lives" IBM Inst. for Busin. Val. 2016 http://www-935.ibm.com/services/us/gbs/thoughtleadership/autoconsumer/

[2] B. Markwalter, "The path to driverless cars", IEEE Cons. Elect. Mag., 6 (2), pp. $125-126,2017$

[3] S. Applin, "Autonomous vehicle ethics: stock or custom?", IEEE Cons. Elect. Mag., 6 (3), pp. $108-110,2017$

[4] B. Frankston, “An alien drove my car: a cabbie from Mars?", IEEE Cons. Elect. Mag., 6 (2), pp. 106 - 107, 2017

[5] W. Lumpkins, "Driverless cars and driverless vacuums: will the madness never end?", IEEE Cons. Elect. Mag., 3 (4), pp. 88 - 91, 2014

[6] Intel, "Technology and computing requirements for self-driving cars", doc. n. 0514/RH/CMD/PDF, 2016

[7] J. Honkanen, "MEMS and sensors in automotive applications on the road to autonomous vehicles: HUD and ADAS", MEMS \& Sensor executive congress, Nov. 2016, Scottsdale, Az, US

[8] A. Munir, "Safety assessment and design of dependable cybercars: for today and the future", IEEE Cons. Elect. Mag., 6 (2), pp.69-77, 2017

[9] R. Thakuer, "Scanning Lidar in advanced driver assistance systems and beyond", IEEE Cons. Elect. Mag, 5 (3), pp. 48-54, 2016

[10] P. Groves, Principles of GNSS, Inertial, and Multisensor Integrated Navigation Systems, Artech House, 2008.

[11] M. Perlmutter, et al., "The future of the MEMS inertial sensor performance, design and manufacturing," DGON Inertial Sen. and Syst. 2016

[12] I. Prikhodko, et al., "In-run bias self-calibration for low-cost MEMS vibratory gyroscopes,” IEEE PLANS 2014, pp. 515-518

[13] J. Collin, "MEMS IMU carouseling for ground vehicles," IEEE Trans. Veh. Technol., 64 (6), pp. 2242-2251, 2015 
[14] M. Schwarz, et al, "The future is MEMS design considerations of microelectromechanical systems at Bosch," IEEE MIXDES 2015, pp. 177-180 [15] Y. K. Thong, et al., "Dependence of inertial measurements of distance on accelerometer noise," Meas. Sci. Technol., 13 (8), 2002

[16] F. Mohd-Yasin et al., "Noise in MEMS," Meas. Sci. Tech., 21, 2010

[17] D. Serrano, "Integrated inertial measurement units using silicon bulkacoustic wave gyroscopes," PhD thesis, Georgia Institute of Technology, 2014. [18] S. Dellea, et al., "In-plane and out-of-plane MEMS gyroscopes based on piezoresistive NEMS detection," J. Microelectromech. Syst., 24 (6), 2015.

[19] M. Hodjat-Shamami, et al., "A dynamically mode-matched piezo electrically transduced high-frequency flexural disk gyroscope," 28th IEEE Int. Con. on Micro Electro Mech. Sys. 2015, pp. 789-792.

[20] J. Cho et al., "High-Q, $3 \mathrm{kHz}$ single-crystal-silicon cylindrical rateintegrating gyro (CING)," IEEE Int. Con. on Micro Elec. Mech. Sys. 2012

[21] "Osram's laser chip for Lidar promises super-short pulses in a smaller package," IEEE Spectrum, Nov. 11, 2016

[22] “Quanergy announces $\$ 250$ solid-state LIDAR for cars, robots, and more," IEEE Spectrum, Jan. 7, 2016

[23] A. Frangi, et al., "Parametric resonance in electrostatically actuated micromirrors," IEEE Trans. Ind. Electron., 64 (2), pp. 1544-1551, 2017.

[24] C. L. Arrasmith, et al., "MEMS-based handheld confocal microscope for in-vivo skin imaging," Opt. Express, 18 (4), pp. 3805-3819, 2010.

[25] U. Hofmann, et al., "Resonant biaxial 7-mm MEMS mirror for omnidirectional scanning," J. Micro/Nanolithography, MEMS, MOEMS, 13, p. 11103,2013

[26] S. Holmstrom, et al., "MEMS laser scanners: a review," Journal of Microelectromechanical Systems, 23 (2), pp. 259-275, 2014.

[27] U. Hofmann, et al., "Wafer-level vacuum-packaged two-axis MEMS scanning mirror for pico-projector application," SPIE MOEMS and Miniaturized Systems XIII, p. 89770A, 2014.

[28] A. Yalcinkaya, et al., "Two-axis electromagnetic microscanner for high resolution displays," J. Microelectromech. Syst., 15 (4), 2006

[29] H.-J. Quenzer, et al., "Piezoelectrically driven translatory optical MEMS actuator with $7 \mathrm{~mm}$ apertures and large displacements," SPIE MOEMS and Miniaturized Systems XIV, p. 93750O, 2015

[30] R. Micheloni et al., Inside NAND Flash memories. Springer-Verlag, 2010

[31] R. Micheloni et al., Inside Solid State Drives (SSDs), Springer series in advanced microelectronics, Springer London, 2012.

[32] JEDEC, "JESD22-A117 document," Oct. 2011.

[33] R. Strenz, "Embedded flash technologies and their applications: Status \& outlook," IEEE IEDM, 2011, pp. 9.4.1-9.4.4.

[34] T. Coughlin, "The memory of cars", IEEE Cons. Elect. Mag., 5 (4), pp. $121-125,2016$

[35] E. Wachmann et al., "ATHENIS 3D: Automotive tested high-voltage and embedded non-volatile integrated SoC platform with 3D technology," IEEE Design Automation and Test in Europe, 2016, pp. 894-899.
[36] M. Thomas et al., "A non-volatile embedded memory for high temperature automotive and high-retention applications," IEEE Int. Symp. on Quality Electronic Design, 2006

[37] C. Zambelli et al., "Characterization of the over-erase algorithm in FN/FN embedded nor flash arrays," IEEE Trans. on Device and Materials Reliability, 15 (4), pp. 529-535, 2015.

[38] C. Zambelli et al., "Exposing reliability/performance tradeoff in nonvolatile memories through erratic bits signature classification," IEEE Trans. on Device and Materials Reliability, 14 (1), pp. 66-73, 2014.

[39] N. Do, "eNVM technologies scaling outlook and emerging NVM technologies for embedded applications," IEEE IMW 2016, pp. 1-4.

[40] H. Wong et al., "Phase Change Memory," Proc. of the IEEE, 98 (12), 2010. [41] D. J. Wouters et al., "Phase-change and redox-based resistive switching memories," Proc. of the IEEE, 103 (8), pp. 1274-1288, 2015.

[42] R. Gaddi et al., "Reliability and performance characterization of a memsbased non-volatile switch," IEEE IRPS 2011, pp. 2G.2.1-2G.2.6.

[43]Y.Zhang, Future wireless networks and information systems, Springer2012

[44] S.-S. Sheu et al., "A 4Mb embedded SLC resistive-RAM macro with 7.2 ns read-write random-access time and 160 ns MLC-access capability," IEEE ISSCC 2011, pp. 200-202.

[45] A. Grossi et al., "Impact of intercell and intracell variability on forming and switching parameters in RRAM arrays," IEEE Trans. on Electron Devices, $62(8)$, pp. 2502-2509, 2015.

[46] C. Zambelli et al., "Phase Change and Magnetic Memories for Solid-State Drive Applications," Proc. of the IEEE, 105 (9), pp. 1790-1811, 2017.

[47] A. Grossi et al., "Reliability and cell-to-cell variability of TAS-MRAM arrays under cycling conditions", IEEE NVMTS 2015, pp. 1-4

[48] S. Brunner at al., "Automotive E/E-architecture enhancements by usage of ethernet TSN", IEEE WISES 2017, pp. 9-13

[49] S. Furst et al., "AUTOSAR for connected and autonomous vehicles: the AUTOSAR adaptive platform", 46th IEEE/IFIP Int. Conf. on Dependable Systems and Networks Workshop, pp. 217-217, 2016

[50] R. Saussard et al., "A robust methodology for performance analysis on hybrid embedded multicore architectures", IEEE MCSoC 2016

[51] P. E. Ross, "CES 2017: Nvidia and Audi Say They'll Field a Level 4 Autonomous Car in Three Years", IEEE Spectrum, 4 Jan 2017

[52] J. Huang, NVIDIA CEO' Keynote, GPU Technology Conference in Europe (GTC2017), Oct 2017, Munich, Germany

[53] S. Saponara et al., "System-level modelling/analysis and LNA design in low-cost automotive technology of a V2X wireless transceiver", IEEE $3^{\text {rd }}$ Int. Forum on Research and Tech. for Society and Industry (RTSI) 2017

[54] P. Guturu, "Explosive wireless consumer demand for network bandwidthfifth generation and beyond", IEEE Cons. Electr. Mag., 6 (2), 2017, pp. 27 - 31 